\title{
ANÁLISIS
}

\section{VISUALIZACIÓN DE LA INFORMACIÓN EN LA DEMOCRATIZACIÓN DE LOS DATOS: PROPUESTAS DESDE EL PERIODISMO Y LA NARRATIVIDAD}

\section{Clàudia Sánchez-Bonvehí y Mireia Ribera}
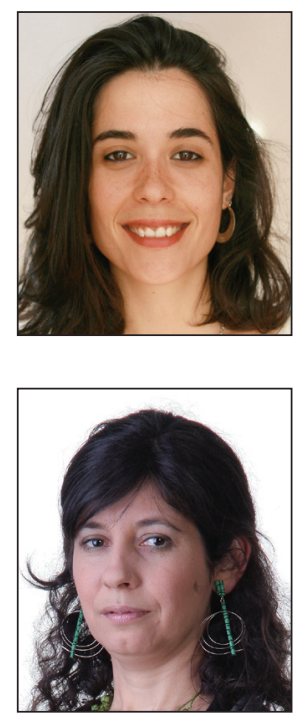

Clàudia Sánchez-Bonvehí es estudiante de doctorado en el programa Información y documentación en la sociedad del conocimiento de la Universitat de Barcelona (UB), licenciada en comunicación audiovisual por la Universitat Pompeu Fabra (UPF) y máster en gestión de contenidos digitales por la UB y UPF. Ha trabajado como periodista en el periódico Regió 7, ha colaborado con el departamento de comunicación y cultura del Instituto Cervantes de Bruselas y como coordinadora del programa Col/laboratori interuniversitari de recursos d'aprenentatge en xarxa (Cirax) en el CBUC. http://orcid.org/0000-0002-5053-7901

claudiasbonvehi@gmail.com

Mireia Ribera es doctora en documentación e ingeniera informática. Es profesora en el Departamento de Biblioteconomía y Documentación de la Universitat de Barcelona (UB). Trabaja en el área de la accesibilidad y usabilidad en lectura digital, y ha impartido la asignatura de visualización de la información en el máster en gestión de contenidos digitales en la UB.

http://orcid.org/0000-0003-1455-1869

ribera@ub.edu

Universitat de Barcelona Melcior de Palau, 140. 08014 Barcelona, España

\section{Resumen}

Se revisa la visualización de la información desde una perspectiva comunicativa, considerándola como una nueva fórmula para la divulgación de datos complejos a un público heterogéneo y no versado en el análisis de datos cuantitativos. Se propone la incorporación de las técnicas de storytelling como herramienta para mantener la atención y conducir la lectura de las visualizaciones, y complementar las dos bases tradicionales de la disciplina: percepción e interacción. La reflexión se acompaña de visualizaciones publicadas en el marco de la comunicación de masas, desde propuestas presentadas por la prensa generalista anglosajona a aplicaciones en línea con el objetivo de aproximar los datos a los ciudadanos.

\section{Palabras clave}

Comunicación, Diseño centrado en el usuario, Visualización de la información, Narración, Democratización de los datos, Visualización para las masas, Infovis.

\section{Title: Information visualization in data democratization: proposals from journalism and storytelling}

\begin{abstract}
Information visualization is reviewed from the communication perspective, which is considered a new formula for disseminating information to a heterogeneous audience of people not specialized in the analysis of quantitative data. The principle of storytelling is incorporated as a tool to hold the attention and direct the reading of the visualizations, and to complement the two traditional bases of the discipline: perception and interaction. The reflection is accompanied by visualizations published in the framework of mass communication, ranging from examples in the English-speaking mainstream press to online applications aimed at making information accessible to the general public.
\end{abstract}

\section{Keywords}

Communication, User-centred design, Information visualization, Storytelling, Data democratization, Visualization for the masses, Infovis.

Sánchez-Bonvehí, Clàudia; Ribera, Mireia (2014). "Visualización de la información en la democratización de los datos: propuestas desde el periodismo y la narratividad". El profesional de la información, mayo-junio, v. 23, n. 3, pp. 311-318. 


\section{Nuevos retos en el acto comunicativo}

La sociedad de la información se caracteriza por la saturación de información y el cambio de roles en el acto comunicativo, que ha dejado hace años de ser unidireccional y estático para estar regido por una diversidad de direcciones y múltiples dinámicas. Asimismo, como consecuencia de los avances y soluciones tecnológicas, la velocidad, el volumen e incluso la diversidad de datos que se generan cada día, crece de forma exponencial y surgen nuevos retos para gestionarlos y dotarlos de sentido, es decir, para convertirlos en información.

Mientras que el ser humano ha desarrollado nuevas habilidades para adaptarse a este nuevo entorno, su atención sigue siendo una capacidad limitada. La nueva situación se caracteriza por una inundación de datos, donde nuestra habilidad cognitiva de dotarlos de sentido y conducir decisiones fiables e informadas, se mantiene relativamente constante (Heer; Kandel, 2012). Como consecuencia de este cuello de botella, el acto comunicativo puede no ocurrir, a pesar de que en él se fundamenta la transmisión de conocimiento.

Para asegurar el equilibro de lo que metafóricamente Hal Varian (Finn, 2011) llama la "obesidad informativa", surge el reto de promover una "dieta informativa equilibrada". Esto exige la calidad de los datos y su explotación de forma entendible y digerible; es decir, que los datos puedan hablar de aquello que representan sin perderse por el camino de la representación. Hablamos de generar significado.

El color, la textura, el movimiento son propiedades preconscientes que llaman la atención de nuestra percepción incluso antes de ser procesadas por nuestro cerebro

Como respuesta a esta necesidad, la visualización de la información emerge para cambiar el proceso comunicativo y devolver estratégicamente el protagonismo ancestral a lo visual. No se trata de vulgarizar los datos, sino de profundizar y aprovechar las ventajas que ofrece la percepción humana y la interacción, con el objetivo de transformar realidades complejas y abstractas en realidades simples.

En nuestra propuesta vamos más allá de los modelos de visualización de información habitualmente establecidos, incorporando los beneficios del storytelling (narratividad) y considerando como público objetivo de las visualizaciones al ciudadano no experto en el análisis de datos ni versado en conceptos estadísticos. La narratividad se plantea como una solución para la creación de visualizaciones que no se dirigen a la comunidad de expertos -en el sentido explicado anteriormente, expertos en análisis de datos y en el manejo de conceptos estadísticos-, y que por el contrario persiguen la divulgación de información, de las historias que cuentan los datos a un público masivo y diverso con motivaciones, intereses y necesidades acentuadamente dispares.

\section{Aproximar los datos a los ciudadanos}

En los últimos años las agencias gubernamentales han intentado presentar los datos con la intención de conseguir el máximo impacto social, pero han fracasado en el intento de aproximar indicadores abstractos (Cukier, 2009), y la mayoría de innovaciones sobre visualización han quedado limitadas al ámbito de la investigación. Como consecuencia, diseñador y usuario final han coincidido en la misma figura: el innovador (Fuhrmann et al., 2005). Se hace evidente pues la necesidad de adaptarse a las características del usuario que no es experto ${ }^{1}$, y por tanto, no presuponer unos conocimientos del fenómeno representado.

La presencia de la visualización de la información (en adelante, infovis) fuera del ámbito académico es cada vez más acentuada. El responsable del departamento de infovis de la OCDE justifica la importancia de la misma como la forma más obvia para transmitir el conocimiento de los expertos a la gente (Cukier, 2009). Diferentes universidades españolas incorporan asignaturas específicas de visualización y han creado nuevos programas de formación en torno a ella². El campo del periodismo ha sido el primero en utilizar técnicas de visualización para narrar las noticias, como una de las técnicas de lo que se ha dado en denominar "periodismo de datos". Con ello se busca llegar a la audiencia más allá del impacto, basándose en la narrativa y el intento de hacer que aquello que era complejo a priori, sea comprensible. Con ello el periodismo ha conseguido convertir la información -previamente basada en datos- en historias.

En este artículo se muestra la importancia de esta aportación, que según las autoras debería convertirse en el tercer pilar básico de la infovis, y se buscan los referentes teóricos que permitan llevarla a su máxima expresión. Finalmente se muestra la necesidad de futuras investigaciones en el área con las técnicas de diseño centrado en el usuario (DCU) para consolidar las propuestas teóricas y los primeros intentos de fusión entre infovis y narratividad.

\section{Dimensiones de la visualización de la información}

La bibliografía tradicional sobre infovis desde sus inicios coincide en considerar dos elementos principales: la teoría de la percepción del ser humano y la explotación de la interacción (Shneiderman, 1996; Card; Mackinlay; Shneiderman, 1999; Spence, 2001; 2007; Segel; Heer, 2010; Ware, 2012).

En este trabajo se incluye una nueva dimensión: la narratividad de la visualización o el storytelling tematizado por Segel y Heer (2010) y presentado como potencial ámbito de desarrollo de las visualización contemporánea por Kosara y Mackinlay (2013). En este apartado procedemos a desglosar estas tres dimensiones que consideramos fundamentales para la concepción, creación y diseño de visualizaciones que se dirigen a usuarios no expertos. Las explicaciones se acompañan de ejemplos en medios de comunicación con el objeto de ilustrar el potencial de las técnicas de visualización para la divulgación de la información. 


\subsection{Percepción: capacidades intrínsecas y compartidas}

El aprovechamiento de los recursos visuales para representar los fenómenos, ya sean datos, relaciones o procesos, es la base que fundamenta la infovis. Como ventajas destacan:

- la amplificación de la cognición humana, puesto que centra la carga del trabajo en el sistema perceptivo en lugar del cognitivo, así como la agrupación de la información y la facilidad de representar una alta densidad de datos en poco espacio;

- reconocimiento de patrones;

- reconocimiento frente a la rememoración (Molich; Nielsen, 1999);

- organización de los datos en relaciones estructurales (Card; Mackinlay; Shneiderman, 1999).

Los datos se codifican de tal forma que los ojos pueden discernir y el cerebro entender (Few, 2013). Para ello los teóricos identifican las propiedades "preatentivas" o "preconscientes", que son aquellas que llaman la atención de nuestra percepción incluso antes de ser procesadas por nuestro cerebro, como color, forma, movimientos, texturas, posición espacial...

Vamos más allá de los modelos de visualización de información habitualmente establecidos, incorporando el storyteIling (narratividad)

Una propiedad es preatentiva cuando se percibe de forma rápida y sin esfuerzos. Ware (2012) habla del fenómeno pop-up, para referirse a la cualidad de emergencia de estas propiedades. Escoger estas características y decidir cuál servirá para codificar cada atributo o dimensión de los datos es clave para la legibilidad de la visualización. Sumándolas se puede favorecer una lectura integrada o una lectura que codifica de forma separada estas dimensiones. El uso combinado del color y la forma para codificar atributos permite una lectura más integrada que el color y el movimiento, por ejemplo.

Según la aportación de Ware, se distinguen tres niveles en el proceso de percepción:

- emergencia de las cualidades preatentivas -forma, color, movimiento, posición espacial-,

- el segundo nivel procesa estas características para favorecer la búsqueda de patrones;

- percepción de objetos.

Un ejemplo del uso de estas propiedades lo encontramos en la figura 1, que representa el grado de respeto a los derechos de los homosexuales en los diferentes estados de EUA. En ella destacamos la utilización de las propiedades preatentivas como el color para identificar los tipos de derecho y la luminancia para mostrar el grado de respeto de estos derechos (menos luminancia-mayor reconocimiento). Por ejemplo en Dakota del Norte se indica con azul claro (más luminoso) el reconocimiento a la adopción para padres solteros; en cambio en Oregon, en azul oscuro, se representa el derecho de adopción a padres solteros y parejas del mismo sexo. Además, a través de la distribución espacial se agrupan los estados por zonas. De este modo en una sola ojeada podemos afirmar que en el noreste del país, casi todos los estados reconocen el matrimonio homosexual (rojo oscuro).

\subsection{Interacción: apropiación e interiorización del tema}

Las nuevas tecnologías permiten dinamizar gráficos hasta ahora estáticos y así posibilitar la exploración de los datos en función de los diferentes criterios del usuario, dejando en segundo plano las visiones fruto de la voluntad del autor. A partir de la puesta en contexto se dota al usuario de un rol interlocutivo.

Las herramientas interactivas de análisis permiten reducir los efectos del big data (Heer; Kandel, 2012), porque permiten al usuario apropiarse de la visualización. En términos de percepción, un diseño eficaz posibilita y vehicula la formulación de hipótesis. La interacción dispone las condiciones para que emerja un proceso iterativo de pregunta-respuesta, paralelo al reconocimiento y la comprensión. Es decir, en contraste con los diseños estáticos, la dimensión de la interacción incorpora el análisis visual, caracterizado por un proceso de creación de visiones, exploración y refinamiento (Heer; Shneiderman, 2012). De esta forma se activa una dinámica de construcción y verificación de hipótesis, circuito necesario para desencadenar que el usuario se apropie del tema representado y entre en diálogo con el diseño visual. 


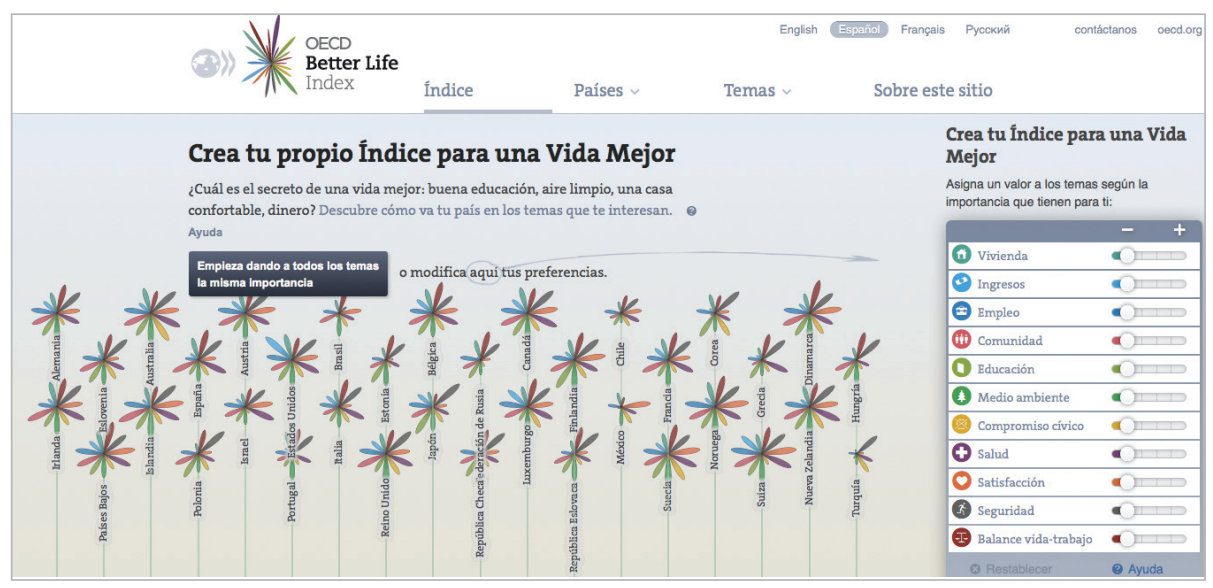

Figura 2. Índice para una vida mejor. (OCDE, 2013).

http://www.oecdbetterlifeindex.org/es

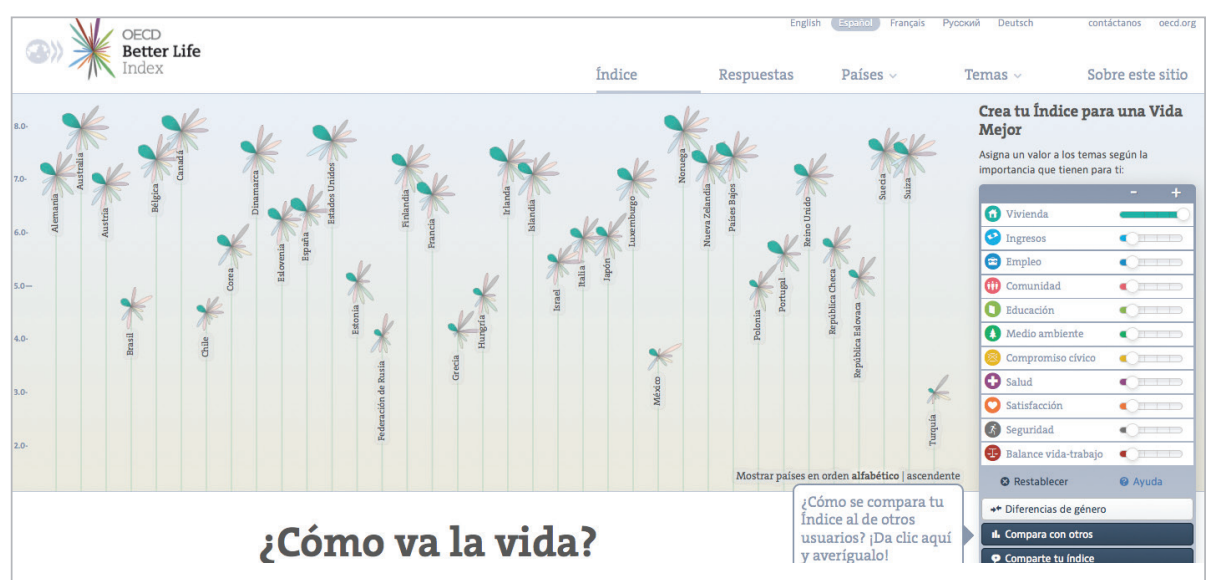

Figura 3. Índice para una vida mejor (OCDE, 2013).

http://www.oecdbetterlifeindex.org/es

La interacción es clave para adaptarse a cada contexto de búsqueda de información donde el entorno se distingue por la cantidad de datos a codificar y la diversidad de objetivos y motivaciones de los usuarios. Por ello no es extraño que Ware (2012) considere que los elementos informativos tienen que ser activos: representar información cuando son necesarios, desaparecer cuando son innecesarios y aceptar el control del usuario para apoyar los procesos de pensamiento.

La interacción permite dotar al usuario de un rol interlocutivo frente a la visualización

Para conseguir esta actividad, Shneiderman (1996) conceptualiza el paradigma de la exploración visual, conocido también como el "mantra" de la búsqueda de información: "primero visión general, zoom y filtro, y luego detalles bajo demanda".

La interfaz tiene que ser lo más transparente posible para facilitar la exploración. La virtud de transparencia dependerá de los conocimientos previos de los usuarios, pero se puede optimizar la visibilidad y aquello que se esconde, como también se puede facilitar la comprensión de las posibilidades de la visualización y de la misma interfaz. Existen recursos que incrementan la potencialidad ${ }^{3}$ de los elementos que integran una visualización, muchos de ellos relacionados con las propiedades preatentivas, que permiten e invitan al usuario a que los objetos sean utilizados para la finalidad para la que han sido concebidos. Además existen otros recursos como la metáfora, que encontramos constantemente cuando navegamos (por ejemplo, cuando con el icono de la mano desplazamos el mapa de la pantalla, o con la lupa nos acercamos a él).

En la visualización creada por la OCDE sobre el Índice para una vida mejor (figura 2), los países se representan por una flor cuyos pétalos corresponden a temas o categorías -vivienda, ingresos, empleo...-. Ya no sólo por el texto que invita al usuario a interactuar, sino también por el panel de la derecha que permite controlar las visiones, el usuario reconoce esta capacidad y la ejecuta. Como resultado de las acciones realizadas por el usuario, la visión presentada cambia. Pongamos por ejemplo que consideramos la "vivienda" como el tema de mayor importancia para nuestro Índice para una vida mejor (figura 3). La visión resultante muestra la organización de los datos y su representación según los criterios del usuario: se resalta el pétalo verde de cada país, que indica la vivienda, y los países cambian de posición. Esta iteración pregunta-respuesta se puede ir repitiendo ya que cada visión resultante puede plantear una duda o una hipótesis que la persona que interactúa va a querer responder, dentro y fuera de la visualización.

\subsection{Storytelling: un hilo conductor para mantener el espectador}

La teoría de la percepción y las posibilidades de interacción han sido estudiadas de forma amplia para desarrollar visualizaciones y han generado conocimiento en torno a la noción de espacio de diseño (Kosara; Mackinlay, 2013). A pesar de estos avances, cuando los datos tienen que ser explicados fuera de la comunidad de expertos es necesario poner en marcha un mecanismo que apele al lector y mantenga su atención. Con la finalidad de gestionar la información proporcionada para vehicular la toma de conocimiento, el hilo conductor emerge como un elemento a tener en cuenta. De forma semejante al espectador que mira una película, al usuario de una visualización la narrativa le revela la información de forma efectiva e intuitiva (Gershon; Page, 2001). 
Esta nueva dimensión que empieza a considerarse en la visualización de la información emerge como un elemento clave para la comunicación de datos. Entender las estructuras narrativas tomadas de otras disciplinas como la literatura o el periodismo, y especialmente en el ámbito de la comunicación audiovisual, puede proporcionar una solución a los nuevos retos comunicativos (Quesenbery; Brooks, 2010). Esta perspectiva incluye la estructura interna del mensaje, una planificación que tiene un guión y múltiples historias que permiten introducir al usuario en la visualización. Incluso se puede considerar inherente a la visualización, pero no siempre ha sido bien gestionada ni tenida en consideración. El mantra de la búsqueda de información de Shneiderman (1996) define, más allá de una guía para facilitar la explotación, un mecanismo que se corresponde con la introducción, el nudo y el desenlace, que caracteriza las fábulas o cuentos propios de gran parte de los géneros populares.

La gran diferencia entre las películas y las visualizaciones por ahora- desde el punto de vista de la narración radica en el control de la progresión, la secuencialidad de los eventos (Segel; Heer, 2010). En función del grado de intervención del autor, del abanico de opciones y control que tiene el usuario, podemos establecer diferentes tipos de narrativa en las visualizaciones. De la excelente categorización de Segel y Heer (2010) destaca la estructura del "vaso de Martini", una combinación de dos esquemas: se inicia con una aproximación guiada por el autor para, más avanzada la exploración, traspasar las riendas al receptor. Como consecuencia del primer contacto guiado por el autor se consigue contextualizar al usuario, mostrar el alcance de la visualización y qué tareas puede llevar a cabo. En la segunda parte, el usuario controla y explora para verificar hipótesis y reformularlas, es decir, poner en marcha la búsqueda interactiva que comentábamos anteriormente. La gestión del tiempo tiene un papel esencial en la representación de datos. Un claro ejemplo sobre el aprovechamiento de la animación para revelar datos lo encontramos en la herramienta Trendalyzer de Gapminder. Se trata de un software de visualización de información para animar estadísticas, desarrollado por Hans Rosling.

http://www.gapminder.org/tag/trendalyzer

El treemap es la representación de un árbol de datos, en el que cada nodo puede tener 0 o más hijos, y se representa por un rectángulo cuyas medidas y color reflejan los valores de los datos. The New York times (figura 4) escoge un treemap para representar la propuesta de Obama sobre la distribución del presupuesto para el 2012. La coordinación entre las historietas y las partes que corresponden a cada una en el treemap se activan al clicar cada lead para hacer un zoom en la parte representada. De esta forma el autor guía o propone diferentes lecturas, que a su vez sirven para mostrar el funcionamiento de la visualización y así actuar también como instrucciones de uso.

las estructuras narrativas tomadas de otras disciplinas como la literatura o el periodismo pueden proporcionar una solución a los nuevos retos comunicativos

\section{Asegurar la correspondencia entre emisor y receptor: el diseño centrado en el usuario (DCU)}

Aunque se ha teorizado alrededor de las teorías de la percepción y la interacción, pocas veces se ha verificado el impacto real en los usuarios. Además, cuando se ha escogido un enfoque que incorpora la persona en el proceso se observa una tendencia a favor de la perspectiva de la interacción persona-ordenador (Lloyd, 2010). Wassink et al. (2009) valoran los pocos estudios que han conseguido traspasar sus resultados a guías de diseño o heurísticos en su revisión sobre las diferentes técnicas de aproximación a los usuarios en el diseño de visualizaciones interactivas destina- 
das a usuarios expertos. La necesidad de disponer de guías para el diseño de visualizaciones con carácter comunicativo y destinadas a una audiencia más heterogénea es aún más patente.

La perspectiva del diseño centrado en el usuario tuvo su origen en el campo militar en los años 50 , se introdujo posteriormente como aproximación para diseñar productos cotidianos, y a mediados de los años 80 tuvo su auge como perspectiva adoptada para el desarrollo de software en el entorno digital. Norman (1988) apunta que la finalidad del diseño centrado en el usuario (DCU en adelante) es conseguir que los productos sean utilizados y comprensibles a partir de conocer los intereses y necesidades del usuario. Para ello el DCU adopta diferentes métodos y técnicas, tanriables que rodean al usuario final -entorno, motivaciones, limitaciones.

\section{El storytelling ayuda a gestionar la infor- mación proporcionada con el fin de ve- hicular la toma de conocimiento}

Como su nombre indica, en el DCU el usuario es el centro que rige las decisiones de diseño y es tangible en todo el proceso puesto que se introduce a lo largo del ciclo de diseño. Destacan técnicas como el prototipado, la técnica personas, escenarios, casos de uso, diseños de interacción, etc.

En 2010 se publica la ISO 9241-2014, para sustituir la ISO 13407 con cierto grado de abstracción (Jokela et al., 2003), y dar respuesta a la cantidad de manuales publicados relativos a este enfoque. La nueva ISO plantea requisitos en lugar de recomendaciones: to cualitativas como cuantitativas para conocer todas las va-

- Entender y especificar el contexto de uso (incluyendo usuarios, tareas y entorno) (análisis).

- Especificar los requerimientos del usuario con suficiente detalle para conducir el diseño e introducir soluciones de diseño (análisis).

- Producir soluciones de diseño que encajen con los requerimientos (diseño).

- Conducir evaluaciones centradas en el usuario de las soluciones de diseño y modificar éste teniendo en cuenta los resultados (evaluación).

Nielsen (1993) además, define el concepto de usabilidad, inherente al DCU, como la suma de cinco atributos, todos ellos cuantificables: facilidad de aprendizaje, eficiencia, facilidad de memorización, tasa de errores y satisfacción.

En el caso de las visualizaciones tratadas en este trabajo, con finalidad divulgativa, será esencial definir la audiencia, por plural que sea, pues para determinar la usabilidad deben definirse los usuarios, sus objetivos concretos y el entorno de uso.

Introducir esta perspectiva para tener presente los diferentes perfiles de usuarios y poder responder a sus requisitos, puede apoyar el diseño de visualizaciones que capten el interés, que respondan a las necesidades y encajen en consultas de tipo informativo o de ocio para la multitud. Se propone a nivel práctico la incorporación de técnicas y metodologías que estudian el usuario, lo definen en diferentes grados de concreción a la vez que lo abstraen para gestionar mejor el proceso de diseño y validación.

A modo de ejemplo del uso de la aplicación de la metodología DCU en el campo de la infovis, la figura 5 presenta un prototipo elaborado para el diseño de una visualización que representa los movimientos migratorios internos en Catalunya. A partir de él se validaron los elementos utilizados para codificar los datos y se verificó si comprendía los recursos adecuados (destacados en color) para llevar a cabo las tareas.

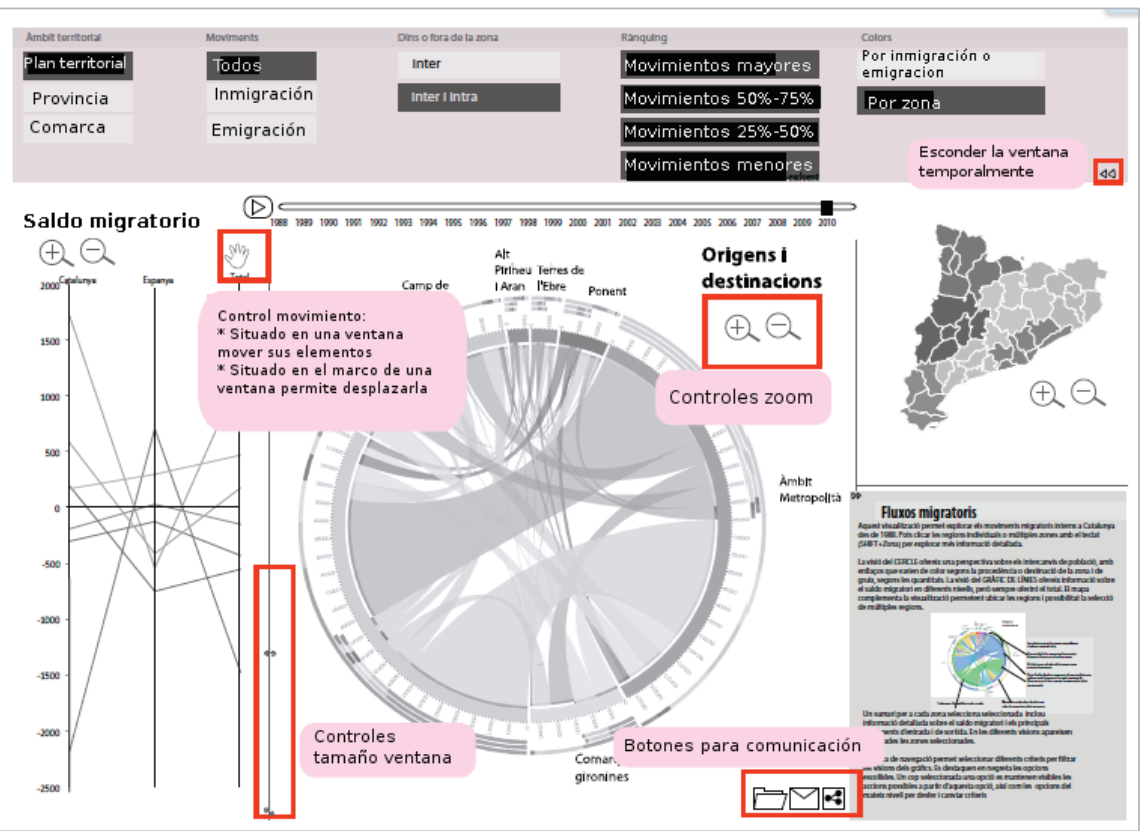

Figura 5. Prototipo de visualización sobre los movimientos migratorios en Catalunya. Controles de zoom, tamaño de las ventanas, opción para esconder las ventanas, control para mover la imagen en el espacio y botones para desarrollar las tareas de comunicación

\section{Conclusiones}

La visualización de la información cumple con los requisitos necesarios para situarse como herramienta privilegiada para la transmisión de conocimiento. Su capacidad representativa, donde prima lo visual por encima de lo textual, permite trabajar de forma rápida y con menor necesidad de procesar en el cerebro. Según los modelos de usabilidad, la percepción directa mejora la eficiencia pues reduce el tiempo de procesamiento, únicamente perceptivo (Card; Mackinlay; Shneiderman, 1999). También veremos que mostrar los datos en visualizaciones cumple con las heurísticas clásicas de usabilidad (Molich; Nielsen, 1990). La visualización se configura a partir de elementos que minimizan el esfuerzo del usua- 
rio, pues es una representación estética y minimalista de los datos, para garantizar que su atención altamente limitada en una marea de estímulos, se centre en los aspectos importantes, la información esencial, la que se quiere comunicar. Ello cumple también con la heurística de prevención de errores al maximizar la captación de atención. Al mismo tiempo, el equilibro entre el titular y la información en detalle puede verse satisfecho gracias a la interacción, dando al usuario un mayor control y libertad sobre los datos mostrados. Los múltiples caminos que éste puede explorar según sus criterios, sus vicisitudes, sus necesidades y sus motivaciones permiten resituarlo en un nuevo espacio privilegiado donde hay lugar para la identificación, la interiorización y, por ende, para la apropiación de la información, ya sea para acumular conocimiento como para ir más lejos aún y desencadenar decisiones.

Es necesario disponer de guías para el diseño de visualizaciones con carácter comunicativo y destinadas a una audiencia heterogénea y no experta

Una visualización interactiva ofrece flexibilidad y eficiencia de uso. Aprovechar estas propiedades para representar la complejidad de los fenómenos puede ampliar la audiencia de contenidos que antes quedaba reducida a una minoría de expertos y a la vez reducir el ruido en la comunicación del mensaje.

Para incrementar las posibilidades de éxito del acto comunicativo, la incorporación de mecanismos narrativos en las visualizaciones se presenta como una nueva dimensión complementaria a la percepción y la interacción, y vendría a completar los anteriores beneficios con ayudas y documentación para guiar al usuario. Aunque se trate de un principio relativamente reciente y poco estudiado en el marco de la infovis, la presencia de visualizaciones que acompañan artículos de divulgación en los medios de comunicación avala su eficacia. Con la tendencia del open data es una dimensión que cobra notoriedad para la reformulación del periodismo.

La propuesta de incluir la narratividad en la infovis debe ser validada pues hay un gran vacío de trabajos que comprueben la eficacia de las visualizaciones, y una necesidad de casos de estudio con resultados extrapolables que permitan la elaboración de guías de diseño. Para articular las dimensiones tratadas en este estudio (percepción, interacción y narrativa), el diseño centrado en el usuario puede proveer un marco de trabajo que garantice el éxito de la comunicación. El DCU permitirá evitar prejuicios sobre los modelos mentales del usuario, definir mejor la audiencia y establecer aquellos elementos que se adecúan mejor a su perfil.

\section{Notas}

1. Nuestro estudio parte de la definición de usuario no experto de Heer et al. (2008) el cual se caracteriza por tener cierto nivel en el manejo de herramientas informáticas o como usuario de internet convencional, que lo utiliza para mantenerse informado pero no es experto en la visualiza- ción de la información. Para reforzar la finalidad comunicativa del estudio, las visualizaciones consultadas por no expertos tendrán lugar en el contexto de utilización proporcionado por Sprague y Tory (2012): fuera del trabajo, con motivaciones casuales, informativas y de ocio.

2. En España en los últimos tres años han nacido programas de Máster en Periodismo de Datos (URJC) y posgrados específicos en Visualización de la información (IDEC, UPF), al mismo tiempo que se han introducido asignaturas de Visualización de datos como en el programa de Máster en Gestión de Contenidos Digitales (UB).

3. Del inglés affordance introducido inicialmente por Gibson y adaptado por Norman (1988).

4. ISO 9241-210:2010. Ergonomics of human-system interaction. Part 210: Human-centered design for interactive systems. TC/SC: ISO/TC 159/SC 4

\section{Bibliografía}

Card, Suart; Mackinlay, Jock; Shneiderman, Ben (1999). Readings in information visualization: using vision to think. Academic Press. Morgan Kaufman Publishers. San Francisco. ISBN: 1558605339

Cukier, Jerome (2009). "Can data visualization improve knowledge and decision-making?" En: Kosara, Robert; Cohe, Sarah; Cukier, Martin. Panel: Changing the world with visualization. IEEE Visualization Conference Compendium. http://kosara.net/papers/2009/Kosara_InfoVisPanel_2009. $p d f$

Few, Stephen (2013) "A more thoughtful but no more convincing view of big data". Perceptual edge. Visual bussines intelligence. A blog by Stephen Few.

http://www.perceptualedge.com/blog/?p=1671

Finn, Holly (2011). "Lunch with Hal Varian”. Think quarterly. The data issue 2011.

http://www.google.co.uk/think/articles/lunch-with-hal. html

Fuhrmann, Sven; Ahonen-Rainio, Paula; Edsall, Robert; Fabrikant, Sara; Koua, Etien; Tobón, Carolina; Ware, Colin; Wilson, Stephanie (2005) "Making useful and usable geovisualization". En: Dykes, Jason; Maceachren, Alan; Kraak, Menno-Jan Design and Evalutaion Issues. Exploring Geovisualization, pp. 553-566. ISBN: 9780080445311 http://dx.doi.org/10.1016/B978-008044531-1/50446-2

Gershon, Nahum; Page, Ward (2001). "What storytelling can do for information visualization". Communications of the ACM, v. 44. n.8, pp. 31-37

http://citeseerx.ist.psu.edu/viewdoc/download?doi=10.1.1. 138. 7625\&rep=rep1\&type $=p d f$ http://dx.doi.org/10.1145/381641.381653

Heer, Jeffrey; Kandel, Sean (2012). "Interactive analysis of big data". En: XRDS: Crossroads. The ACM magazine for students, v. 19, n. 1, pp 50-54.

http://dx.doi.org/10.1145/2331042.2331058

Heer, Jeffrey; Shneiderman, Ben (2012). "Interactive dynamics for visual analysis". Communications of the ACM, v. 55, n. 4, pp. 45-54. 
http://idl.cs.washington.edu/files/2012-InteractiveDynamicsCACM.pdf

http://dx.doi.org/10.1145/2133806.2133821

Heer, Jeffrey; Van-Ham, Frank; Caprendale, Sheelagh; Weaver, Chris; Isenberg, Petra (2008). "Creation and collaborations: engaging new audiences for information visualizations" Information visualization LNCS 4950. Springer-Verlag, pp. 92-133.

http://dx.doi.org/10.1007/978-3-540-70956-5_5

Jokela, Timo; livari, Netta; Matero, Juha; Karukka, Minna (2003). "The standard of user-centered design and the standard definition of usability: analyzing ISO 13407 against ISO 9241-11" En: Proceedings of the Latin American conference on Human-computer interaction (CLIHC'03). ACM, New York, pp. 53-60.

http://mcom.cit.ie/staff/Computing/prothwell/HCl/papers/ ISO\%20Standards\%20Analysis\%202003\%20Jokela.pdf http://dx.doi.org/10.1145/944519.944525

Kosara, Robert; Mackinlay, Jock (2013). "Storytelling: next step for visualization" En: IEEE Computer Society, v. 46, n. 5, pp. 44-50.

http://kosara.net/papers/2013/Kosara_Computer_2013.pdf http://dx.doi.org/10.1109/MC.2013.36

Lloyd, David (2010). Evaluating human-centered approaches to geovisualization. City University London.

Molich, Rolf; Nielsen, Jakob (1990). "Improving a humancomputer dialogue". Communications of the ACM, v. 33, n. 3, pp. 338-348.

http://cpe.njit.edu/dlnotes/cis/cis732_447/cis732_2r.pdf http://dx.doi.org/10.1145/77481.77486

Nielsen, Jakob (1993) Usability engineering. Boston, Massachusets: Morgan Kaufmann Publishers, San Francisco. ISBN: 0125184050

Norman, Donald (1988). The psychology of everyday things.
Basic Books. ISBN: 0465067093

Quesenbery, Whitney; Brooks, Kevin (2010). Storytelling for user experience. Ronsenfeld Media. ISBN: 1933820039

Segel, Edward; Heer, Jeffrey (2010) "Narrative visualization: telling stories with data". En: IEEE Transactions on Visualization and Computer Graphics, pp. 1139-1148.

http://vis.stanford.edu/files/2010-Narrative-InfoVis.pdf

Shneiderman, Ben (1996) "The eyes have it: a data type taxonomy for information visualizations" En: Proceedings of the 1996 IEEE Symposium on Visual Languages, pp. 336-343. http://www.cs.umd.edu/ ben/papers/Shneiderman1996eyes. $p d f$

http://dx.doi.org/10.1109/VL.1996.545307

Spence, Robert (2001). Information visualization. ACM, New York. ISBN: 9780201596267

Spence, Robert (2007). Information visualization: design for interaction. Pearson/Prentice Hall, Second edition. ISBN: 9780132065504

Sprague, David; Tory, Melanie (2012). "Exploring how and why people use visualization in casual contexts: modeling user goals and regulated motivations". Information visualization, v. 11, n. 2, pp. 106-203.

http://dx.doi.org/10.1177/1473871611433710

Ware, Colin (2012). Information visualization: perception for design. Morgan Kaufmann Publishers. Third edition, San Francisco. ISBN: 0123814642

Wassink, Ingo; Kulyk, Olga; Van-Dijk, Betsy; Van-der-Veer, Gerrit; Van-der-Vet, Paul (2009). "Applying user-centered approach to interactive visualisation design". En: Trends in interactive visualisation, advanced information and knowledge processing, pp. 175-199. http://doc.utwente.nl/64840/1/WassinkKulyk.pdf http://dx.doi.org/10.1007/978-1-84800-269-2_8

\section{Suscripción EPI sólo online}

Pensando sobre todo en los posibles suscriptores latinoamericanos, ya no es obligatorio pagar la suscripción impresa de EPI para acceder a la online.

EPI se ofrece a instituciones en suscripción "sólo online" a un precio considerablemente más reducido $(101,65+21 \%$ IVA euros/año = 123 euros/año), puesto que en esta modalidad no hay que cubrir los gastos de imprenta ni de correo postal.

Para los suscriptores particulares, el precio de la sucripción "sólo online" es de $62+21 \%$ IVA euros/año $=75$ euros/año. 